\title{
Synthesis and Characterization of Drug-Loaded Poly(E-caprolactone)/Silica Hybrid Nanofibrous Scaffolds
}

\author{
Kwan-Ha Shin, ${ }^{1}$ Young-Hag Koh, ${ }^{1,2}$ and Hyoun-Ee Kim ${ }^{3}$ \\ ${ }^{1}$ Department of Dental Laboratory Science and Engineering, Korea University, Seoul 136-703, Republic of Korea \\ ${ }^{2}$ Department of Orthopaedics, Korea University Medical Center, Guro Hospital, Seoul 152-703, Republic of Korea \\ ${ }^{3}$ Department of Materials Science and Engineering, Seoul National University, Seoul 151-742, Republic of Korea
}

Correspondence should be addressed to Young-Hag Koh; kohyh@korea.ac.kr

Received 19 July 2013; Revised 27 September 2013; Accepted 27 September 2013

Academic Editor: Raghavendra Hegde

Copyright (C) 2013 Kwan-Ha Shin et al. This is an open access article distributed under the Creative Commons Attribution License, which permits unrestricted use, distribution, and reproduction in any medium, provided the original work is properly cited.

\begin{abstract}
We produced drug-loaded poly( $\varepsilon$-caprolactone) (PCL)/silica hybrid nanofibrous scaffolds with various silica sol contents $(0 \mathrm{vol} \%$, $10 \mathrm{vol} \%, 15 \mathrm{vol} \%$, and $20 \mathrm{vol} \%$ ) using electrospinning and examined their physicochemical properties, mechanical properties, drug release behavior, and in vitro biocompatibility to evaluate their potential application for guided bone regeneration (GBR). The loading efficiency of tetracycline hydrochloride $(\mathrm{TCH})$ drug was remarkably enhanced by hybridizing the PCL solution with the silica sol. All produced hybrid scaffolds had a highly nanofibrous structure, in which the silica phase was homogeneously hybridized with the PCL polymer, while preserving their intrinsic characteristics. This led to considerable increases in hydrophilicity and mechanical properties (e.g., ultimate tensile strength and elastic modulus). In addition, the release rate and cumulative maximum amounts of the TCH from the hybrid scaffolds significantly increased with increasing the silica content, while all produced hybrid nanofibrous scaffolds showed good biocompatibility assessed by in vitro cell tests.
\end{abstract}

\section{Introduction}

Fundamentally, extracellular matrix (ECM) plays a crucial regulatory role in guiding cells attachment, growth, migration and differentiation as well as formation of new tissues [1, 2]. This encouraged materials scientists and engineers to design and create new biomaterials that can mimic the micro/nanostructure and chemical composition of the native ECM [3]. One of the most promising approaches for this goal is to use electrospinning (ES), which can create a nanofibrous structure with high porosity and surface-to-volume ratio. This biomimetic nanofibrous structure with biocompatible surfaces can provide a favorable environment for guided bone regeneration (GBR) [4-8]. In addition, nanofibrous scaffolds can deliver drugs in a controlled manner [9-14], which is expected to prevent damages caused by infection, thereby accelerating bone healing process when used as a bone scaffold $[15,16]$.

As a scaffolding material, poly( $\varepsilon$-caprolactone) (PCL), one of the most widely used biodegradable polymers, has been extensively examined owing to its proper strength and flexibility [17-20]. However, PCL polymer has low stiffness, hydrophobic nature, and limited cell affinity [8]. Thus, considerable effort has been made to incorporate bioactive inorganic particles into PCL nanofibers for improving the biocompatibility in vitro and in vivo [21-23]. More recently, sol-gel derived silica has gained increasing interest as an alternative inorganic phase, since it can be homogenously hybridized with biodegradable polymers at the molecular level [24-29]. These organic/inorganic hybrids can more closely mimic the chemical composition and nanostructure of the native bone ECM, leading to significantly enhanced mechanical properties and biocompatibility [30].

In this study, we produced drug-loaded PCL/silica hybrid nanofibrous scaffolds using ES and examined their physicochemical properties, mechanical properties, drug release behavior, and in vitro biocompatibility to evaluate their potential application for GBR. For this goal, PCL solutions were directly hybridized with various amounts of the silica sol ( 0 vol\%, 10 vol\%, 15 vol\%, and $20 \mathrm{vol} \%$ in relation to PCL) 
containing tetracycline hydrochloride $(\mathrm{TCH})$ used as a drug. The nanofibrous structure, chemical composition, and chemical structure were characterized by a range of analysis tools, including field emission scanning electron microscope (FESEM), energy dispersive spectroscopy (EDS), and attenuated total reflection Fourier transform infrared spectroscopy (ATR-FTIR). The mechanical properties were examined by tensile strength tests. The release behavior of the TCH from the PCL/silica hybrid nanofibrous scaffolds was examined. The in vitro biocompatibility was evaluated in terms of proliferation of preosteoblast cells.

\section{Materials and Methods}

2.1. Starting Materials. Unless specified otherwise, all reagents were purchased from Sigma-Aldrich (Sigma Aldrich, St. Louis, MO, USA).

\subsection{PCL/Silica Hybrid Mixture Preparation. A PCL solution} was prepared by dissolving $12 \mathrm{~g}$ PCL $\left(M_{n}=80,000 \mathrm{~g} / \mathrm{mol}\right)$ in $100 \mathrm{~mL}$ of tetrahydrofuran (THF) at $40^{\circ} \mathrm{C}$ using a magnetic stirrer. Separately, a silica sol was synthesized through sol-gel process at room temperature by mixing tetraethyl orthosilicate $\left(\mathrm{Si}\left(\mathrm{OC}_{2} \mathrm{H}_{5}\right)_{4}\right.$; TEOS), distilled water, and ethanol at a molar ratio of $1: 4: 1$ with the assistance of $1 \mathrm{~N} \mathrm{HCl}$ as a catalyst using a magnetic stirrer for $2 \mathrm{~h}$. Subsequently, predetermined amounts of the silica sol ( $0 \mathrm{vol} \%, 10 \mathrm{vol} \%$, $15 \mathrm{vol} \%$, and $20 \mathrm{vol} \%$ in relation to PCL) were added to the PCL solutions and vigorously mixed by magnetic stirring for $30 \mathrm{~min}$, after which, tetracycline hydrochloride $(\mathrm{TCH})$ used as a drug with a concentration of $20 \mathrm{mg} / \mathrm{mL}$ was added to the PCL/silica hybrid mixtures, followed by magnetic stirring for $2 \mathrm{~h}$ at room temperature. The viscosity of the $\mathrm{PCL} /$ silica hybrid mixtures was measured using a viscometer (Brookfield Dial Reading Viscometer, Hwashin Instrument Co. Ltd., Republic of Korea).

2.3. Electrospinning Process. The prepared PCL/silica hybrid mixtures containing TCH were loaded into $10 \mathrm{~mL}$ syringes. Electrospinning was conducted at a flow rate of $2 \mathrm{~mL} / \mathrm{h}$ and a tip-target distance of $10 \mathrm{~cm}$, while a range of applied voltages $(7-17 \mathrm{kV})$ were applied to achieve a uniform nanofibrous structure depending on the silica sol content ( $0 \mathrm{vol} \%, 10 \mathrm{vol} \%$, $15 \mathrm{vol} \%$, and $20 \mathrm{vol} \%)$. PCL/silica hybrid nanofibrous scaffolds produced were removed carefully from the mandrel and kept at room temperature in ambient condition more than 3 days to completely remove the solvents. For comparison purposes, dense PCL/silica hybrid films were also prepared by spin coating technique.

2.4. Nanofibrous Structure Evaluation. The morphology of the TCH-loaded PCL/silica hybrid nanofibrous scaffolds with various silica sol contents ( 0 vol $\%, 10 \mathrm{vol} \%, 15 \mathrm{vol} \%$, and 20 vol\%) was examined by field emission scanning electron microscopy (FE-SEM; JSM-6330F, JEOL, Japan). The diameters of the hybrid nanofibers were roughly measured from the SEM images. The chemical composition and structure of the hybrid nanofibrous scaffolds were characterized by energy dispersive spectroscopy (EDS) attached to the FE-SEM and attenuated total reflection Fourier transform infrared spectroscopy (ATR-FTIR, Nicolet 6700, Thermo Scientific., USA), respectively. The nanostructure of the hybrid nanofibers was observed more closely using transmission electron microscopy (TEM) (JEM-2000EXII, JEOL, Japan) after the PCL/silica hybrid mixtures were directly electrospun onto a copper grid coated with an amorphous carbon film. Energy dispersive X-ray spectroscopy (EDS) analysis was carried out during the TEM observations to confirm the existence of silica in the hybrid nanofibers.

The final content of the silica phase in the hybrid nanofibrous scaffolds with various silica sol contents ( 0 vol\%, $10 \mathrm{vol} \%, 15 \mathrm{vol} \%$, and $20 \mathrm{vol} \%)$ was determined by thermogravimetric analysis (TGA; TA Instruments, New Castle, DE, USA). The hybrid nanofibrous scaffolds were heated up to $700^{\circ} \mathrm{C}$ at a heating rate of $10^{\circ} \mathrm{C} / \mathrm{min}$ in a flowing nitrogen atmosphere. The weight losses of the specimens during the tests were monitored and used to calculate the final silica contents.

2.5. Hydrophilicity Measurement. The hydrophilicity of the TCH-loaded PCL/silica hybrid nanofibrous scaffolds with various silica sol contents ( $0 \mathrm{vol} \%, 10 \mathrm{vol} \%, 15 \mathrm{vol} \%$, and $20 \mathrm{vol} \%$ ) was evaluated using water contact angle measurements using an OCA15 contact angle analyzer (Dataphysics Co, Germany). A distilled water droplet size of $\sim 2 \mu \mathrm{L}$ from a syringe was placed carefully onto the surface of the hybrid nanofibrous scaffolds at room temperature. After a period of 20 seconds, the contact angle was recorded. The mean value and standard deviation (SD) were calculated through testing at five different positions on the same sample.

2.6. Mechanical Properties Test. The mechanical properties of the TCH-loaded PCL/silica hybrid nanofibrous scaffolds were evaluated using tensile strength tests. The specimens with dimensions of $7.5 \mathrm{~mm} \times 10 \mathrm{~mm} \times 0.02 \mathrm{~mm}$ were tested at a crosshead speed of $1 \mathrm{~mm} / \mathrm{min}$ using a screw-driven load frame (Oriental Testing Machine Co, Republic of Korea) at a temperature of $\sim 37^{\circ} \mathrm{C}$ [31]. The stress versus strain responses of the specimens during the tensile strength tests were recorded. The tensile strength and strain at failure were obtained from stress versus strain curves. The elastic modulus was determined from the slope of the initial linear portion of the curves. Six specimens were tested to obtain the mean and standard deviation for each hybrid nanofibrous scaffold.

2.7. Drug Release Test. The drug release behavior of the TCH-loaded PCL/silica hybrid nanofibrous scaffolds with various silica sol contents ( 0 vol\%, $10 \mathrm{vol} \%, 15 \mathrm{vol} \%$, and $20 \mathrm{vol} \%$ ) was examined. The specimens were immersed in $6 \mathrm{~mL}$ of phosphate buffered saline (PBS) solution ( $\mathrm{pH} 7.4$ ) and then placed in a water bath at $37^{\circ} \mathrm{C}$ for up to $48 \mathrm{~h}$. At selected intervals, $3 \mathrm{~mL}$ aliquots were withdrawn and analyzed using an ultraviolet (UV) spectrophotometer (V630; JASCO, Japan) at an optimal wavelength of $355 \mathrm{~nm}$. An equal volume of fresh medium was added to the incubation solution. The release amount of the TCH was calculated 


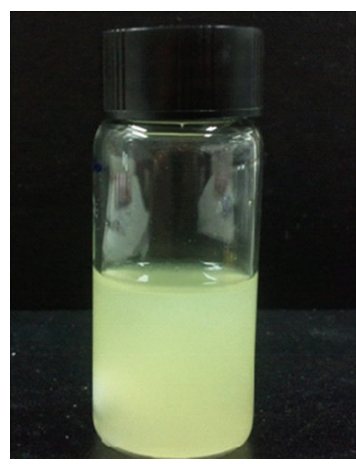

(a)

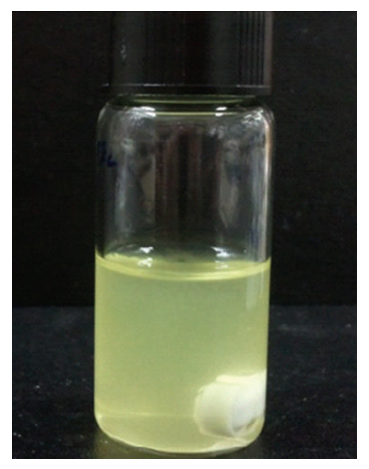

(b)

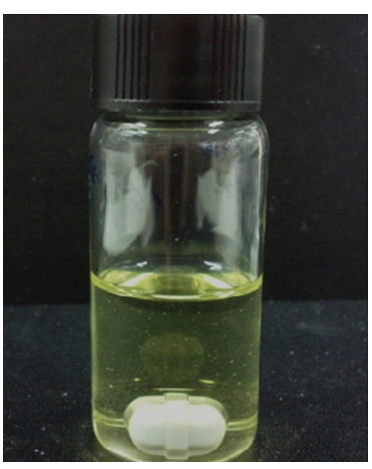

(c)

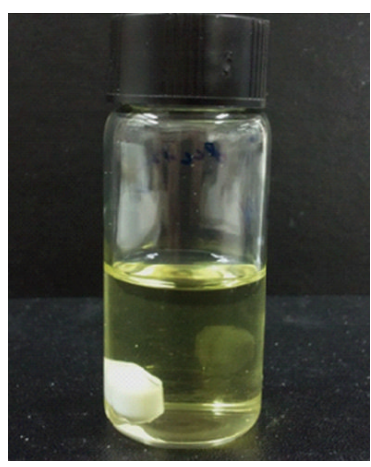

(d)

FIgure 1: Typical optical image of the TCH-loaded PCL/silica hybrid mixtures with various silica sol contents $(0 \mathrm{vol} \%, 10 \mathrm{vol} \%, 15 \mathrm{vol} \%$, and 20 vol $\%)$.

from an absorbance-concentration calibration curve, which was constructed over the concentration range from 1 to $100 \mu \mathrm{g} / \mathrm{mL}$, and the cumulative TCH release was represented after being normalized to its initial amount in the scaffolds, obtained by multiplying the weight of the preobtained nanofibrous scaffold by the theoretical weight percentage of TCH. The drug-release test was performed in triplicate, and data were reported as mean \pm standard deviation (SD).

2.8. In Vitro Biocompatibility Evaluation. The in vitro biocompatibility of the TCH-loaded PCL/silica hybrid nanofibrous scaffolds with various silica sol contents ( $0 \mathrm{vol} \%$, $10 \mathrm{vol} \%, 15 \mathrm{vol} \%$, and $20 \mathrm{vol} \%)$ was evaluated using a preosteoblast cell line (MC3T3-E1; ATCC, CRL-2593, Rockville, MD, USA). The MC3T3-E1 cells were cultured in Duebecco's modified eagle medium (DMEM: Welgene Co., Ltd., Seoul, Republic of Korea) supplemented with 5\% fetal bovine serum (FBS) in a humidified incubator in an atmosphere containing $5 \% \mathrm{CO}_{2}$ at $37^{\circ} \mathrm{C}$. Prior to the cell seeding, the specimens with dimensions of $1 \mathrm{~mm} \times 1 \mathrm{~mm}$ were sterilized under ultraviolet (UV) irradiation for $30 \mathrm{~min}$ and soaked in $70 \%$ ethanol for $4 \mathrm{~h}$. Subsequently, the cells were plated on the specimens at a density of $3 \times 10^{4}$ cells $/ \mathrm{mL}$ for cell attachment and a density of $1 \times 10^{4}$ cells $/ \mathrm{mL}$ for cell proliferation and then cultured in a humidified incubator in an atmosphere containing $5 \% \mathrm{CO}_{2}$ at $37^{\circ} \mathrm{C}$ for up to 2 days.

The morphologies of the attached cells on the hybrid nanofibrous scaffolds after $6 \mathrm{~h}$ of culturing were examined by confocal laser scanning microscopy (CLSM; C1 PLUS, Nikon, Tokyo, Japan). For these CLSM observations, the cells on the specimens were fixed in 4\% paraformaldehyde in PBS for $10 \mathrm{~min}$, washed in PBS, permeabilized with $0.1 \%$ Triton X-100 in PBS for 7 min, washed in PBS and stained with fluorescent phalloidin for $30 \mathrm{~min}$. The cell nuclei were counterstained with DAPI for $5 \mathrm{~min}$. The stained specimens were placed on a cover slide, and the cell morphology was observed.

The cell viability and growth of the hybrid nanofibrous scaffolds after 1 and 2 days of culturing were examined using an MTS (methoxyphenyl tetrazolium salt) assay with 3(4, 5-dimethylthiazol-2-yl)-5-(3-carboxymethoxyphenyl)-2(4-sulfophenyl)-2H-tetrazolium (MTS, Celltiter 96 Aqueous
Promega, Madison, WI, USA) for mitochondrial reduction. After 1 and 2 days of culturing, the specimens were washed by PBS and reacted with the MTS solution for $4 \mathrm{~h}$. The quantity of the formazan product, which would be directly proportional to the number of living cells in the culture, was measured by the absorbance at $490 \mathrm{~nm}$ using a microplate reader (Model 550; Biorad, Hercules, CA, USA). Four species for each condition were tested to obtain mean and standard deviation. The continued cell proliferation on the $\mathrm{TCH}$ loaded PCL/silica hybrid nanofibrous scaffolds was also evaluated after 7 days of culturing.

2.9. In Vitro Apatite-Forming Ability Evaluation. The in vitro apatite-forming ability of the TCH-loaded PCL/silica hybrid nanofibrous scaffolds with various silica sol contents ( $0 \mathrm{vol} \%$, $10 \mathrm{vol} \%, 15 \mathrm{vol} \%$, and $20 \mathrm{vol} \%$ ) was characterized by soaking in a simulated body fluid (SBF) solution for 7 days. The specimens were immersed in the SBF at a concentration of $3 \mathrm{mg} \mathrm{mL}^{-1}$ in polyethylene bottles with the initial $\mathrm{pH}$ of the solutions being kept at 7.40. The specimens were then placed inside an incubator at a controlled temperature of $37^{\circ} \mathrm{C}$ for 7 days, extracted, washed three times with ethanol, and then dried at $37^{\circ} \mathrm{C}$ for $12 \mathrm{~h}$. The formation of the apatite layer on the surface of the hybrid scaffold was examined using FE-SEM and EDS analyses.

2.10. Statistical Analysis. The statistical significance of differences in the data was analyzed using a one-way ANOVA test based on the software Origin Lab 8.0 (Microcal Co, USA). $P$ values $<0.05\left(^{*}\right)$ were considered significant.

\section{Results and Discussion}

3.1. Hybridization of PCL-Silica-TCH. One of the most important steps for the production of PCL/silica hybrid nanofibrous scaffolds with a controlled release of drug is to prepare homogenous hybrid mixtures. Figure 1 shows a typical optical image of TCH-loaded PCL/silica hybrid mixtures with various silica sol contents $(0 \mathrm{vol} \%, 10 \mathrm{vol} \%$, $15 \mathrm{vol} \%$, and $20 \mathrm{vol} \%)$. The TCH-loaded PCL solution was 


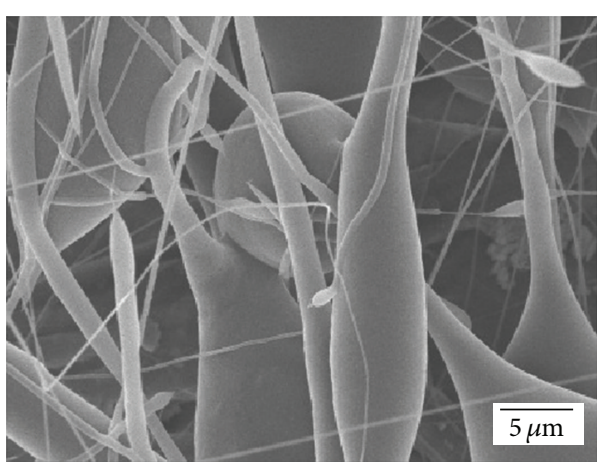

(a)

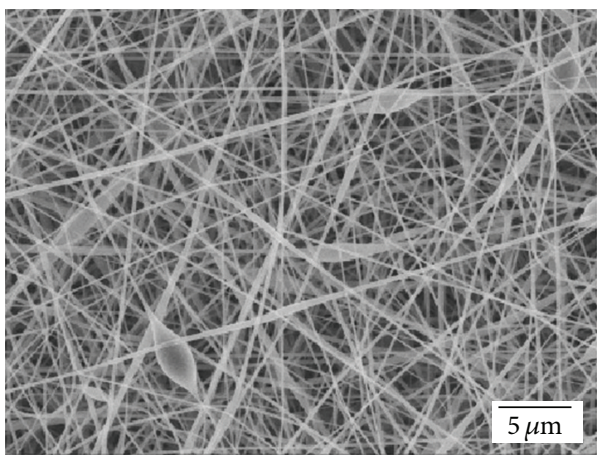

(c)

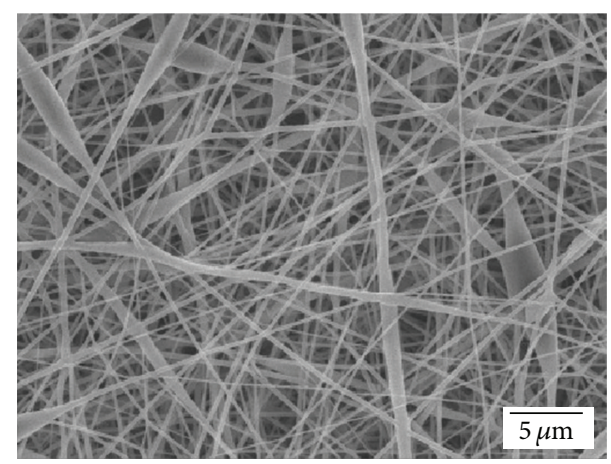

(b)

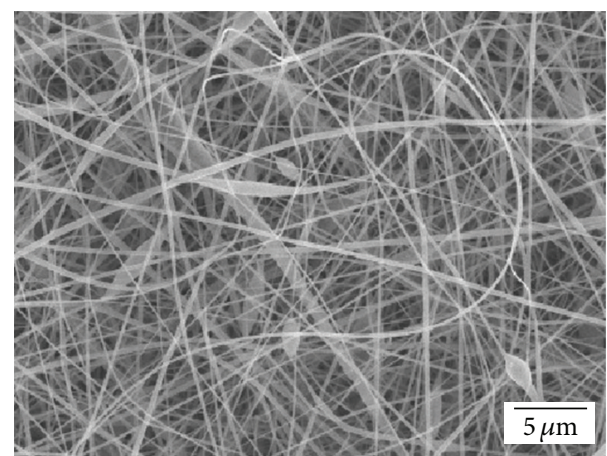

(d)

FIGURE 2: Typical FE-SEM images of the TCH-loaded PCL/silica hybrid nanofibrous scaffolds with various silica sol contents of (a) 0 vol\%, (b) $10 \mathrm{vol} \%$, (c) $15 \mathrm{vol} \%$, and (d) $20 \mathrm{vol} \%$.

cloudy. Conversely, the TCH-loaded PCL/silica hybrid mixtures turned clear with increasing the silica content. This finding suggests that the hybridization of the PCL solution with the silica sol is highly beneficial to the loading of the $\mathrm{TCH}$ drug, which is presumably due to a mesoporous structure of sol-gel derived silica [32].

\subsection{Morphology and Nanostructure of Hybrid Nanofibrous} Scaffolds. Figures 2(a)-2(d) show typical morphologies of the TCH-loaded PCL/silica hybrid nanofibrous scaffolds with various silica sol contents $(0 \mathrm{vol} \%, 10 \mathrm{vol} \%, 15 \mathrm{vol} \%$, and 20 vol\%). The TCH-loaded PCL nanofibrous scaffold showed a relatively nonuniform fibrous structure (Figure 2(a)), containing large quantities of very thick and flattened fibers. In addition, the $\mathrm{TCH}$ drug tended to be segregated from the PCL solution during electrospinning process, resulting in a nonuniform distribution of the TCH drug in the PCL nanofibers. On the other hand, all produced PCL/silica hybrid scaffolds with various silica sol contents $(10 \mathrm{vol} \%$, $15 \mathrm{vol} \%$, and $20 \mathrm{vol} \%$ ) showed a highly uniform, nanofibrous structure (Figures 2(b)-2(d)). This finding suggests that the loading of the TCH drug can be significantly enhanced by hybridizing the PCL solution with the silica sol.

The diameter of the hybrid nanofibers decreased with increasing the initial silica sol content, as shown in Figures 3(A)-3(D). This was mainly attributed to a decrease in the viscosity of the $\mathrm{PCL} /$ silica hybrid mixtures. The measured viscosities of the $\mathrm{PCL} /$ silica hybrid mixtures with silica contents of $0 \mathrm{vol} \%, 10 \mathrm{vol} \%, 15 \mathrm{vol} \%$, and $20 \mathrm{vol} \%$ were 250 , 33,22 , and $18 \mathrm{mPa} \cdot \mathrm{s}$, respectively.

The hybridization of the PCL polymer with silica phase was evaluated by TEM, as shown in Figures 4(a) and 4(b). The PCL/silica hybrid nanofibrous scaffold with a highest silica content of $20 \mathrm{vol} \%$ showed that the PCL polymer was uniformly hybridized with the silica phase on the nanoscale (Figure 4(a)). In addition, EDS analysis confirmed the presence of the silica phase in the hybrid nanofibers (Figure 4(b)). This finding suggests that PCL polymer can be uniformly hybridized with silica and TCH drug, allowing for the production of $\mathrm{TCH}$-loaded hybrid nanofibrous scaffolds with a controlled micro-/nanostructure.

3.3. Chemical Composition. The presence of the silica phase in the TCH-loaded PCL/silica hybrid nanofibrous scaffolds with various silica sol contents ( 0 vol\%, $10 \mathrm{vol} \%, 15 \mathrm{vol} \%$, and $20 \mathrm{vol} \%$ ) was confirmed by EDS analyses, as shown in Figures 5(a)-5(d). The PCL nanofibrous scaffold showed peaks associated with $\mathrm{C}$ and $\mathrm{O}$ elements (Figure 5(a)), while all produced PCL/silica hybrid nanofibrous scaffolds showed an additional strong peak corresponding to Si element (Figures $5(\mathrm{~b})-5(\mathrm{~d})$ ). The relative intensities of the peaks associated with $\mathrm{Si}$ and $\mathrm{O}$ elements increased with increasing the silica content.

The final content of the silica phase in the PCL/silica hybrid nanofibrous scaffolds with various silica sol contents ( $0 \mathrm{vol} \%, 10 \mathrm{vol} \%, 15 \mathrm{vol} \%$, and $20 \mathrm{vol} \%$ ) was calculated by 


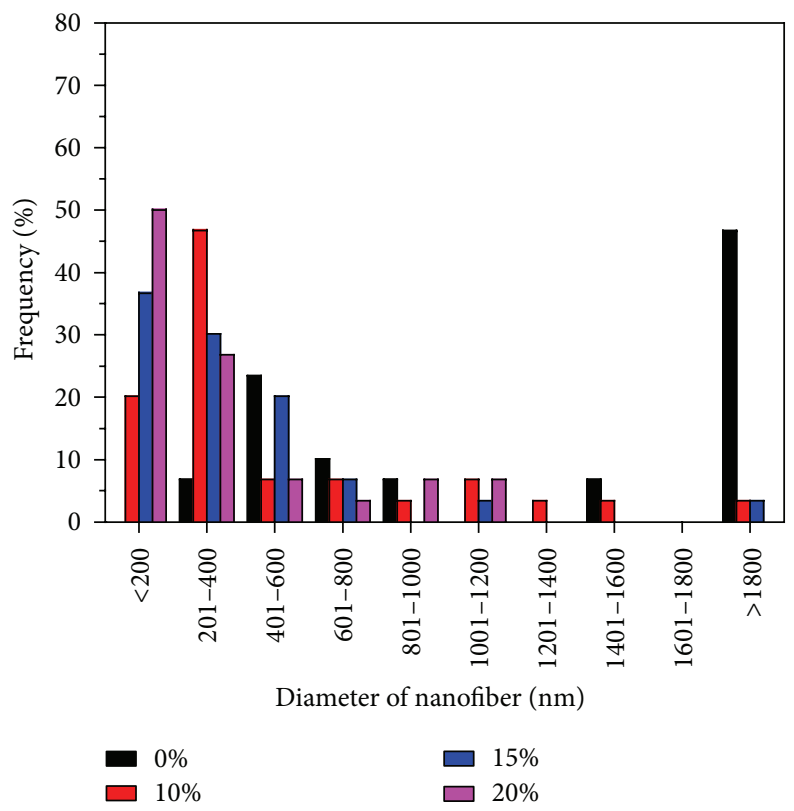

Figure 3: The diameter distributions of the PCL/silica hybrid nanofibers with various silica sol contents ((A) 0 vol\%, (B) 10 vol \%, (C) 15 vol\%, and (D) $20 \mathrm{vol} \%$ ).

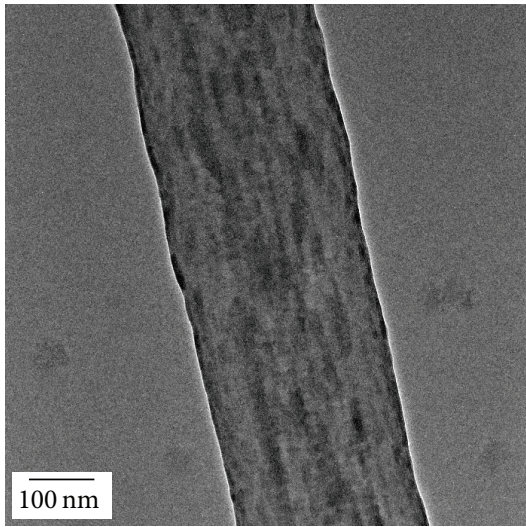

(a)

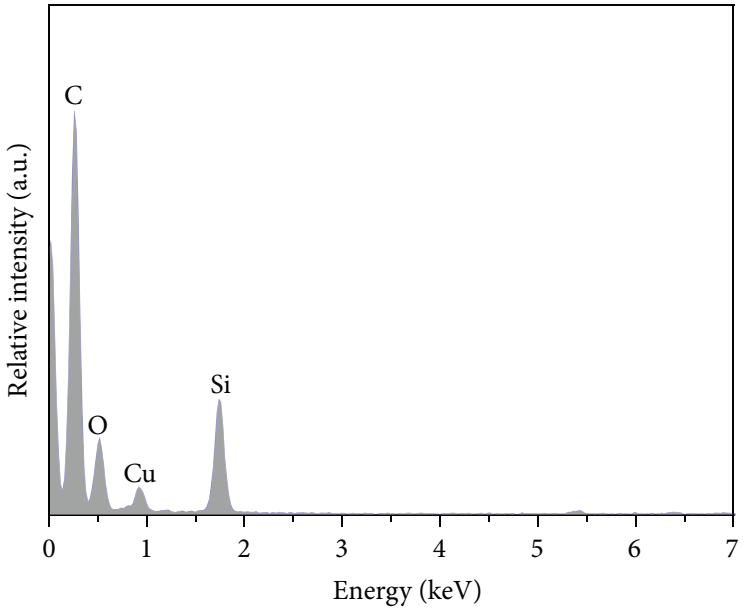

(b)

FIGURE 4: (a) TEM image of the PCL/silica hybrid nanofibers produced with a silica content of $20 \mathrm{vol} \%$ and (b) EDS spectrum of the nanofiber.

TGA analysis, as shown in Figures 6(A)-6(D). All specimens showed similar thermal behaviors only associated with thermal decomposition of the PCL polymer with negligible thermal change of the silica phase. More specifically, significant weight loss was observed at $\sim 380^{\circ} \mathrm{C}$, attributed to thermal decomposition of the PCL polymer [33, 34]. The final content of the silica phase was $14.5 \mathrm{wt} \%, 21.0 \mathrm{wt} \%$, and $27.6 \mathrm{wt} \%$ for the initial silica sol contents of $10 \mathrm{vol} \%, 15 \mathrm{vol} \%$, and 20 vol\%, respectively, which corresponded well to the originally intended values (i.e., $15.04 \mathrm{wt} \%, 22.91 \mathrm{wt} \%$, and $28.10 \mathrm{wt} \%)$. This finding suggests that the silica phase can be homogeneously hybridized with the PCL polymer without any considerable loss during process.
3.4. Chemical Structure. The chemical structure of the TCHloaded PCL/silica hybrid nanofibrous scaffolds with various silica sol contents ( $0 \mathrm{vol} \%, 10 \mathrm{vol} \%, 15 \mathrm{vol} \%$, and $20 \mathrm{vol} \%$ ) was investigated by ATR-FTIR to examine a chemical interaction between the PCL and silica phases, as shown in Figures 7(A)-7(D). The PCL nanofibrous scaffold showed typical bands of PCL polymer, namely, two dominant absorption peaks at 1725 and $1180 \mathrm{~cm}^{-1}$, corresponding to the stretching vibrations of the carboxyl $(\mathrm{C}=\mathrm{O})[35]$ and ether groups $(\mathrm{C}-$ $\mathrm{O}-\mathrm{C}$ ) [36], respectively (Figure $7(\mathrm{~A})$ ). On the other hand, all produced PCL/silica hybrid nanofibrous scaffolds with various silica contents (10 wt $\%, 15 \mathrm{wt} \%$ and $20 \mathrm{wt} \%)$ showed additional bands at approximately 1078, 795 and $453 \mathrm{~cm}^{-1}$, 


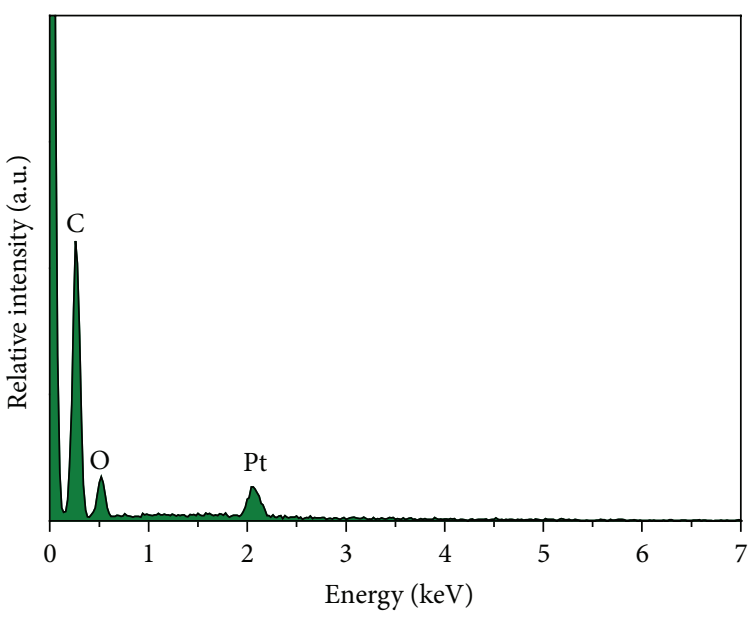

(a)

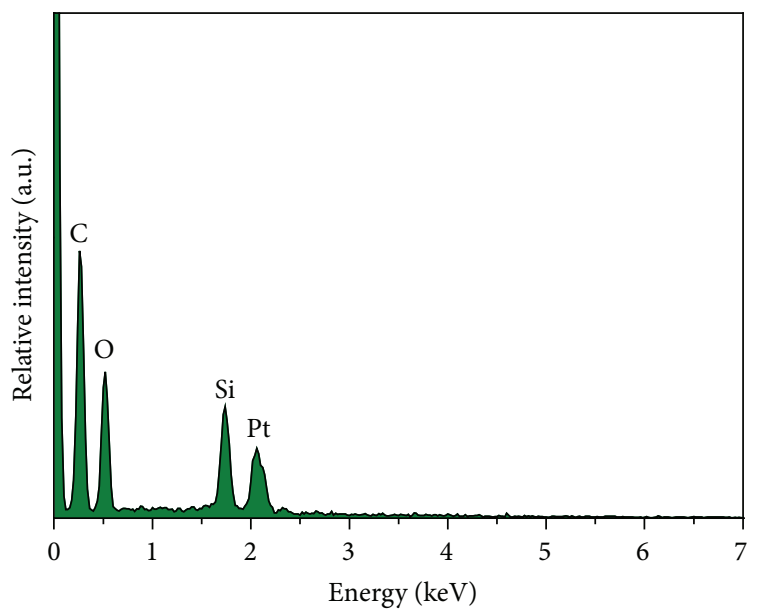

(c)

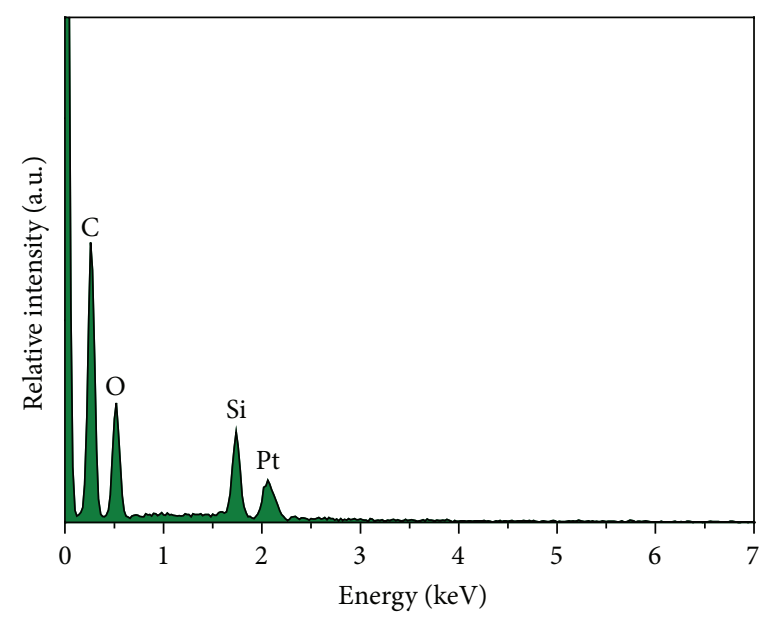

(b)

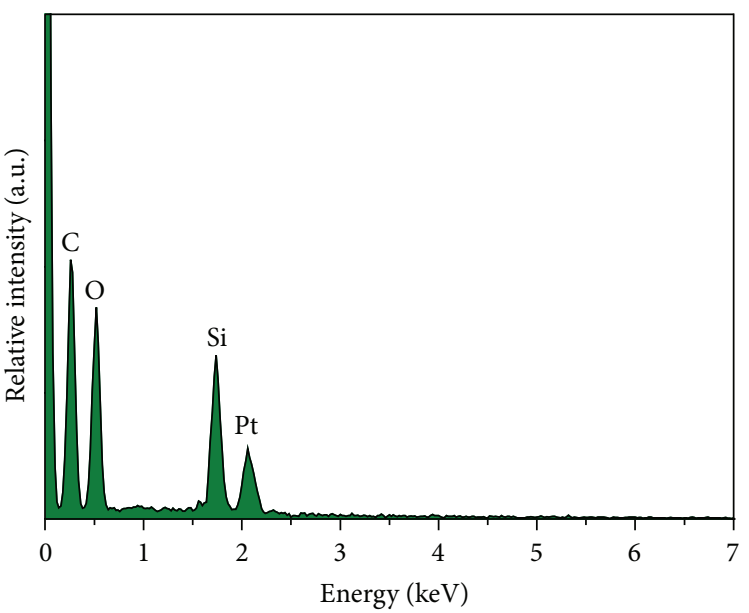

(d)

FIGURE 5: Typical EDS spectrums of the TCH-loaded PCL/silica hybrid nanofibrous scaffolds with various silica sol contents of (a) 0 vol\%, (b) $10 \mathrm{vol} \%$, (c) $15 \mathrm{vol} \%$, and (d) $20 \mathrm{vol} \%$.

which are representative of the various vibration modes of $\mathrm{Si}$ $\mathrm{O}-\mathrm{Si}$ bonds $[37,38]$.

No noticeable band shift was observed, suggesting that the intrinsic characteristics of the PCL and silica phases are preserved.

3.5. Hydrophilicity. The hydrophilicity of the TCH-loaded $\mathrm{PCL} /$ silica hybrid nanofibrous scaffolds with various silica contents ( $0 \mathrm{vol} \%, 10 \mathrm{vol} \%, 15 \mathrm{vol} \%$, and $20 \mathrm{vol} \%$ ) was evaluated by measuring the incident contact angle, as shown in Figure 8 . The PCL and PCL/silica hybrid nanofibrous scaffold with an initial silica content of $10 \mathrm{wt} \%$ showed relatively high contact angles of $99 \pm 7^{\circ}$ and $101 \pm 3^{\circ}$, respectively. However, the PCL/silica hybrid nanofibrous scaffold with an initial silica content of $15 \mathrm{wt} \%$ showed a much lower contact angle of $64 \pm 3^{\circ}$. In addition, the PCL/silica hybrid nanofibrous scaffold with an initial silica content of $20 \mathrm{wt} \%$ showed a contact angle near zero; that is, a water droplet quickly spread and penetrated the nanofibrous structure, as is often the case with nanoporous PCL/silica xerogel membranes [39].
However, the contact angle of the dense PCL/silica hybrid films prepared by spin coating technique did not change much, that is, $59 \pm 6^{\circ}, 48 \pm 6^{\circ}, 51 \pm 4^{\circ}$, and $56 \pm 6^{\circ}$ for the hybrid films with silica contents of 0 vol $\%, 10 \mathrm{vol} \%, 15 \mathrm{vol} \%$, and 20 vol\%, respectively. This was presumably due to the phase separation of the silica phase from the PCL polymer, which differed from $\mathrm{PCL} /$ silica composites with a uniform distribution of the silica phase in the PCL polymer that showed significantly enhanced hydrophilicity [39]. However, it is reasonable to suppose that the nanofibrous structure is one of the critical factors for enhancing the hydrophilicity of the PCL/silica hybrid nanofibrous scaffolds.

3.6. Mechanical Properties. The mechanical properties of the TCH-loaded PCL/silica hybrid nanofibrous scaffolds were evaluated at a temperature of $\sim 37^{\circ} \mathrm{C}$ using tensile strength tests. Figures 9(A)-9(D) show typical stress versus strain responses of the hybrid nanofibrous scaffolds with various silica sol contents ( 0 vol\%, 10 vol $\%, 15$ vol\% and 20 vol\%). The PCL nanofibrous scaffold showed a typical ductile 


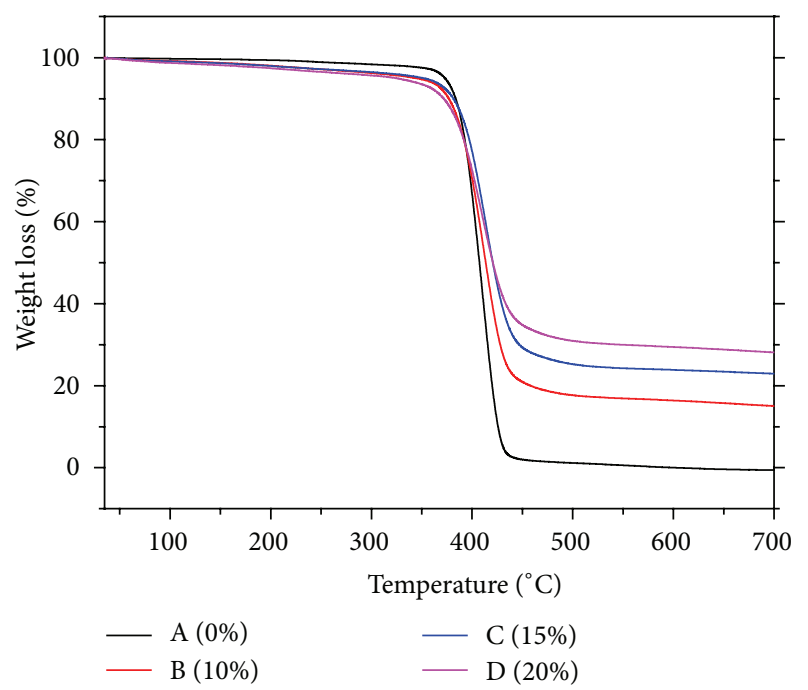

FIgURE 6: Weight losses of the TCH-loaded PCL/silica hybrid nanofibrous scaffolds with various silica sol contents of (A) $0 \mathrm{vol} \%$, (B) $10 \mathrm{vol} \%$, (C) $15 \mathrm{vol} \%$, and (D) $20 \mathrm{vol} \%$ as a function of temperature.

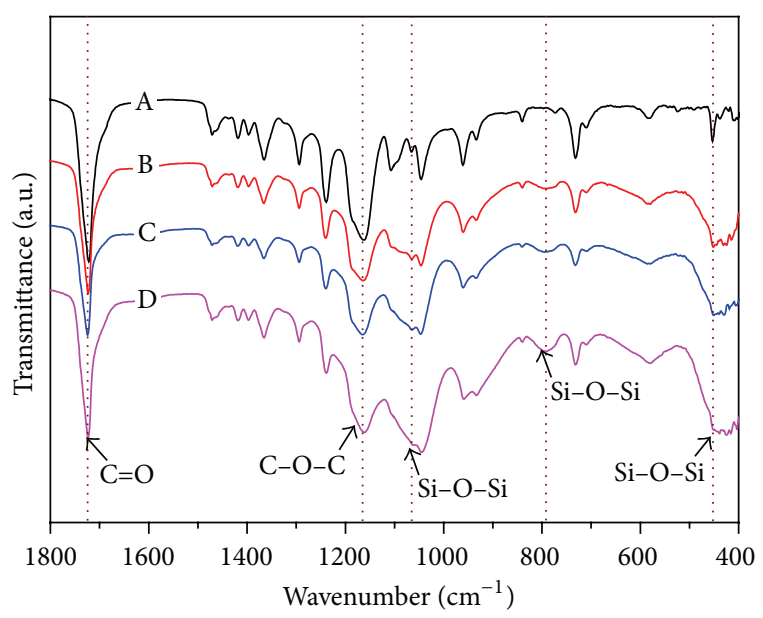

FIgURE 7: Typical ATR-FTIR spectrums of the TCH-loaded PCL/silica hybrid nanofibrous scaffolds with various silica sol contents of (A) $0 \mathrm{vol} \%$, (B) $10 \mathrm{vol} \%$, (C) $15 \mathrm{vol} \%$, and (D) $20 \mathrm{vol} \%$.

fracture behavior of ductile polymers (Figure 9(A)), but relatively low mechanical properties due to its nonuniform nanofibrous structure. On the other hand, the PCL/silica hybrid nanofibrous scaffolds with initial silica sol contents of $10 \mathrm{vol} \%$ and $15 \mathrm{vol} \%$, showed much higher maximum stresses with rapid decreases in stress after fracture (Figures $9(B)$ and $9(C))$. However, a higher silica content of $20 \mathrm{vol} \%$ considerably decreased the maximum stress and strain at failure (Figure 9(D)).

Table 1 summarizes the ultimate tensile strength, elastic modulus, and strain at failure of the TCH-loaded PCL/silica hybrid nanofibrous scaffolds with various silica sol contents ( $0 \mathrm{vol} \%, 10 \mathrm{vol} \%, 15 \mathrm{vol} \%$, and $20 \mathrm{vol} \%$ ). The hybrid nanofibrous scaffold with a silica sol content of $15 \mathrm{vol} \%$ showed

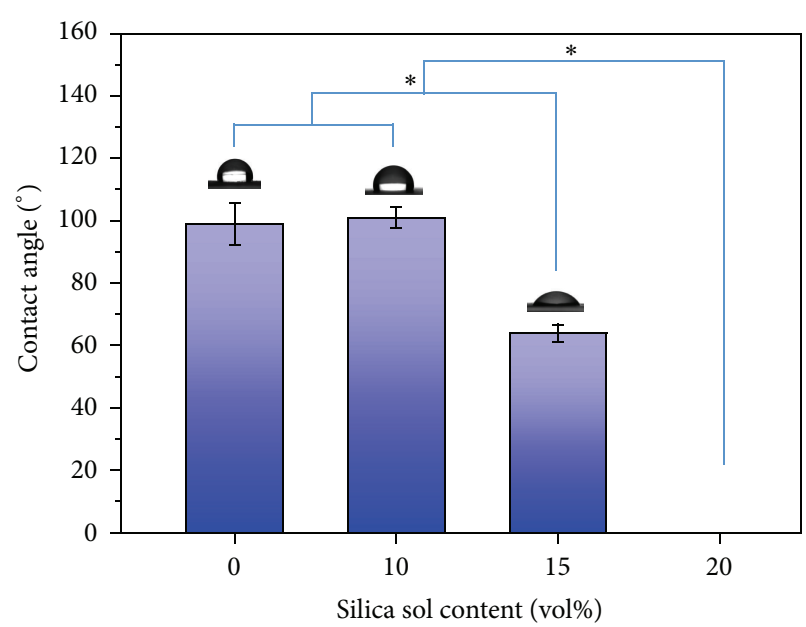

Figure 8: Contact angles of the TCH-loaded PCL/silica hybrid nanofibrous scaffolds as a function of the initial silica sol content. The insets show optical images of the water droplets on the hybrid nanofibrous scaffolds.

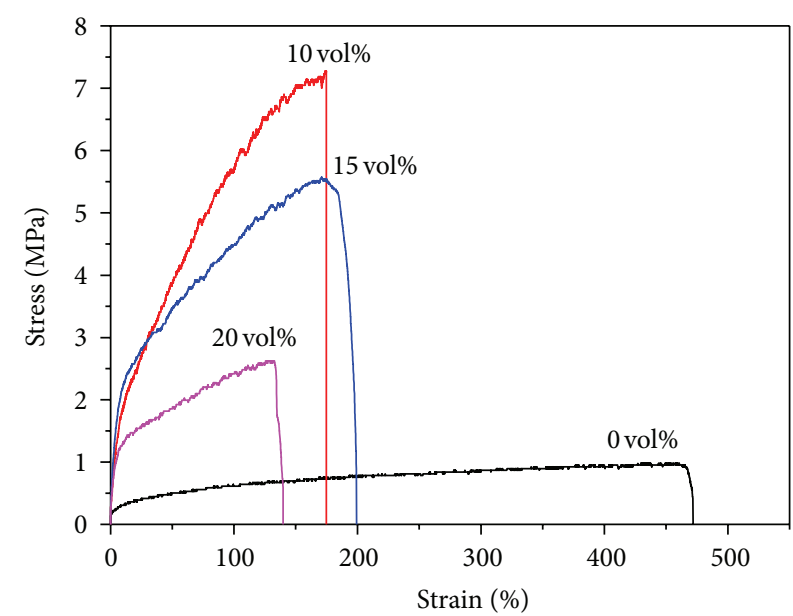

Figure 9: Typical stress versus strain responses of the $\mathrm{TCH}$ loaded PCL/silica hybrid nanofibrous scaffolds with various silica sol contents ((A) $0 \mathrm{vol} \%$, (B) $10 \mathrm{vol} \%$, (C) $15 \mathrm{vol} \%$, and (D) $20 \mathrm{vol} \%$ ).

TABLE 1: Ultimate tensile strength, elastic modulus, and strain at failure of the TCH-loaded PCL/silica hybrid nanofibrous scaffolds with various silica sol contents $(0 \mathrm{vol} \%, 10 \mathrm{vol} \%, 15 \mathrm{vol} \%$, and 20 vol\%).

\begin{tabular}{lccc}
\hline $\begin{array}{l}\text { Silica sol } \\
\text { content (vol\%) }\end{array}$ & $\begin{array}{c}\text { Ultimate tensile } \\
\text { strength (MPa) }\end{array}$ & $\begin{array}{c}\text { Elastic } \\
\text { modulus (MPa) }\end{array}$ & $\begin{array}{c}\text { Strain at } \\
\text { failure (\%) }\end{array}$ \\
\hline 0 & $1.1 \pm 0.2$ & $1.3 \pm 0.3$ & $453 \pm 96$ \\
10 & $7.4 \pm 0.4$ & $17.5 \pm 1.7$ & $193 \pm 20$ \\
15 & $5.5 \pm 0.3$ & $19.8 \pm 2.9$ & $200 \pm 35$ \\
20 & $2.8 \pm 0.5$ & $21.0 \pm 2.2$ & $97 \pm 23$ \\
\hline
\end{tabular}

reasonably high ultimate tensile strength of $5.5 \pm 0.3 \mathrm{MPa}$, elastic modulus of $19.8 \pm 2.9 \mathrm{MPa}$ and strain at failure of $200 \pm$ $35 \%$. This finding suggests that the mechanical properties of the PCL nanofibrous scaffold can be significantly improved 


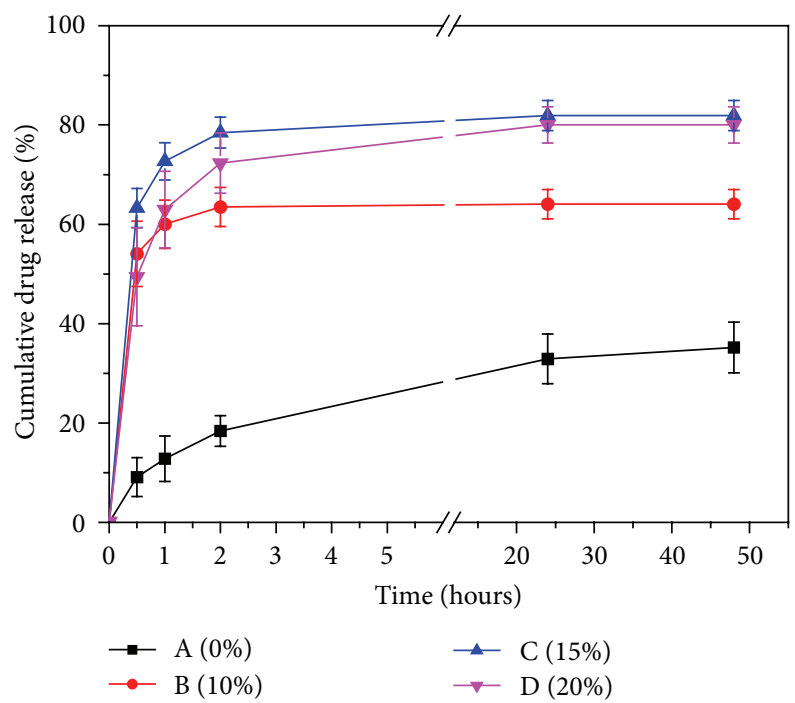

Figure 10: Cumulative drug-release profiles of the TCH-loaded PCL/silica hybrid nanofibrous scaffolds with various silica sol contents of (A) 0 vol\%, (B) $10 \mathrm{vol} \%$, (C) $15 \mathrm{vol} \%$, and (D) $20 \mathrm{vol} \%$.

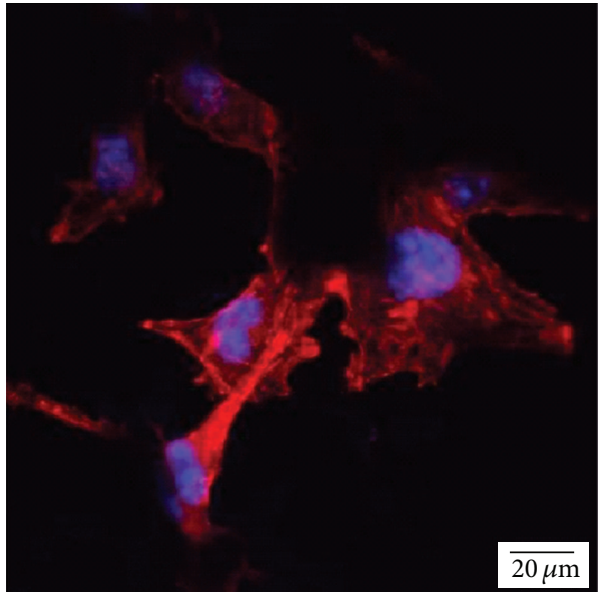

(a)

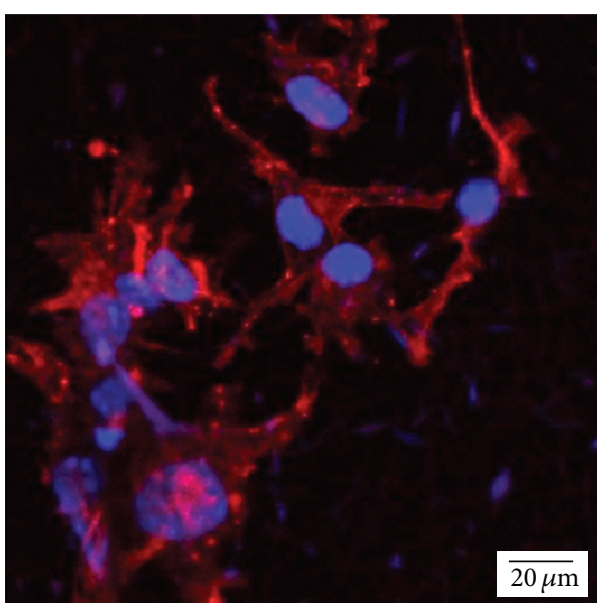

(c)

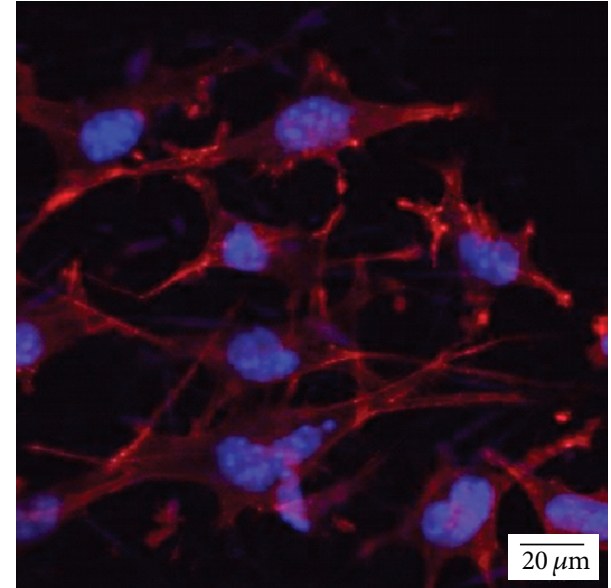

(b)

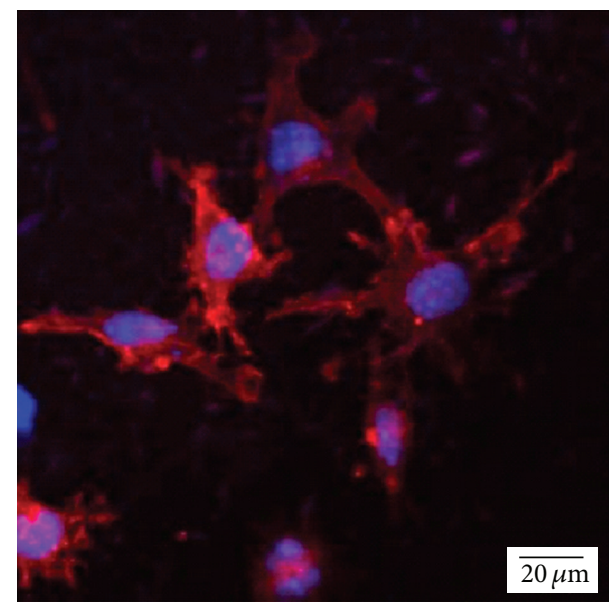

(d)

FIGURE 11: Typical CLSM images of the MC3T3-E1 cells attached on the TCH-loaded PCL/silica hybrid nanofibrous scaffolds with various silica sol contents of (a) $0 \mathrm{vol} \%$, (b) $10 \mathrm{vol} \%$, (c) $15 \mathrm{vol} \%$, and (d) $20 \mathrm{vol} \%$ after $6 \mathrm{~h}$ of culturing. 


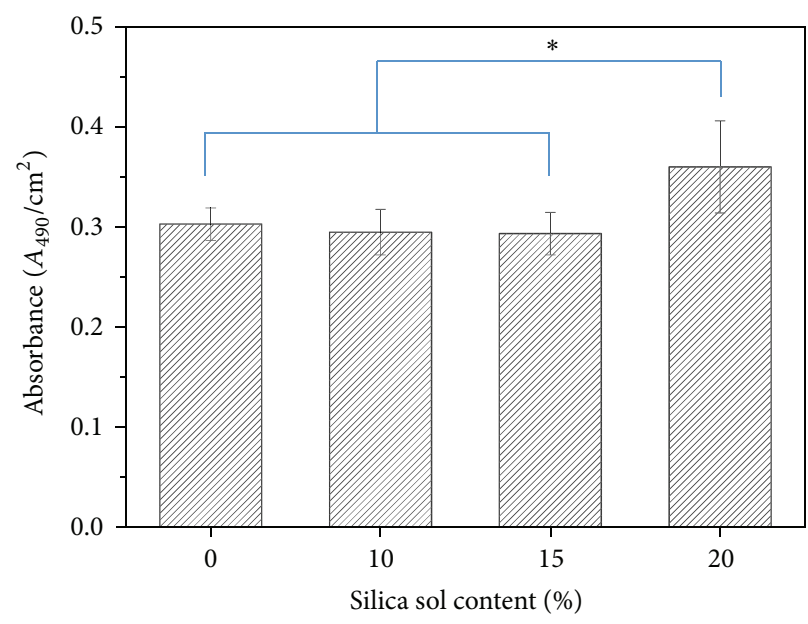

(a)

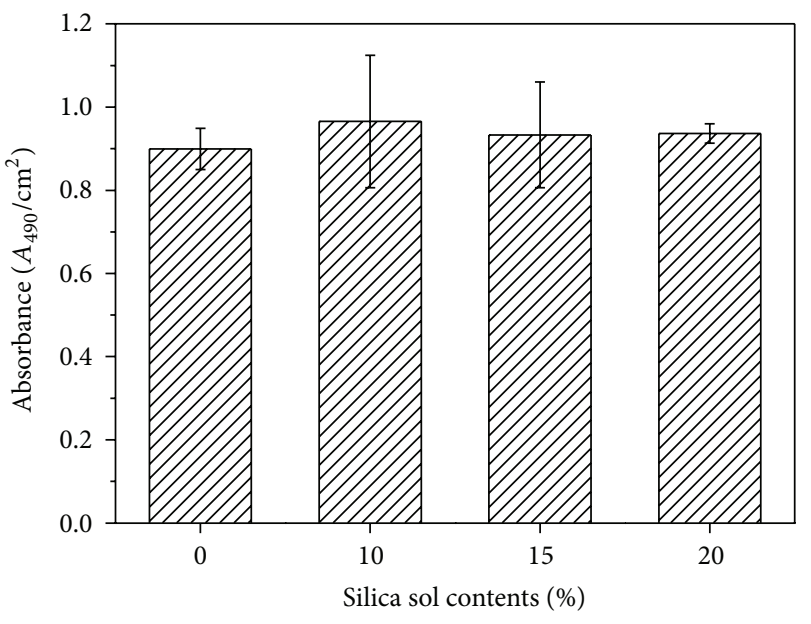

(b)

FIgure 12: Cell viabilities of the MC3T3-E1 cells on the TCH-loaded PCL/silica hybrid nanofibrous scaffolds (a) after 1 day and (b) 2 days of culturing.

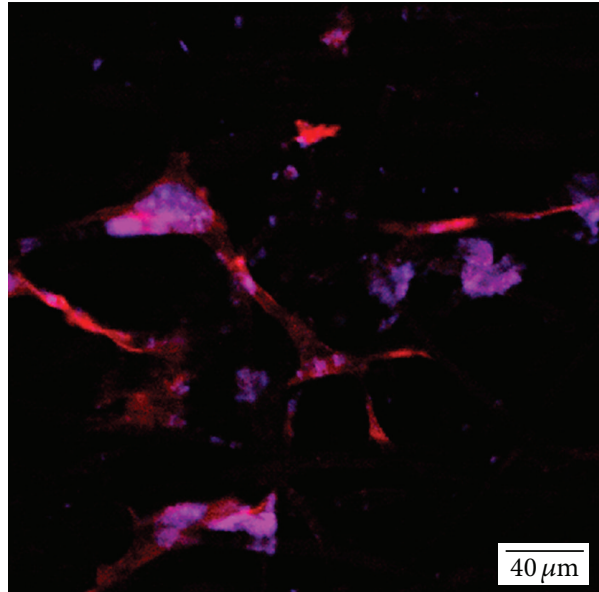

(a)

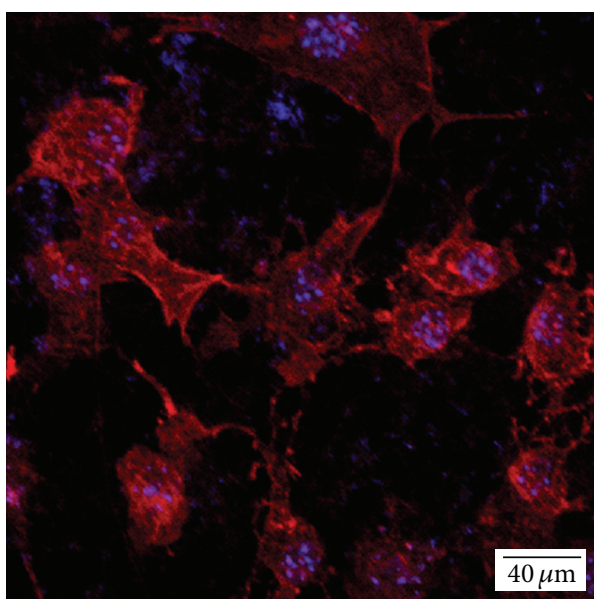

(c)

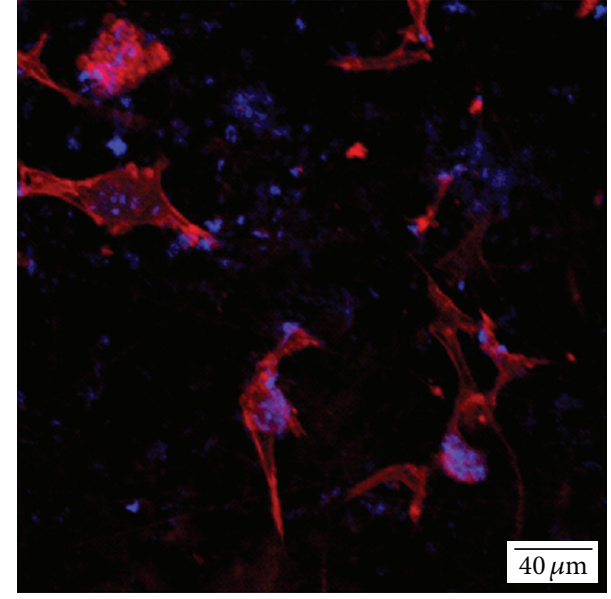

(b)

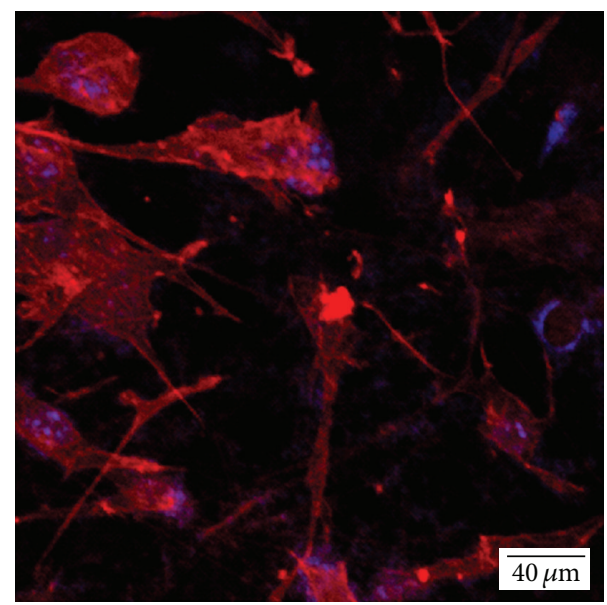

(d)

FIGURE 13: CLSM images of the MC3T3-E1 cells on the TCH-loaded PCL/silica hybrid nanofibrous scaffolds with various silica sol contents of (a) 0 vol\%, (b) $10 \mathrm{vol} \%$, (c) $15 \mathrm{vol} \%$, and (d) 20 vol\% after 7 days of culturing. 


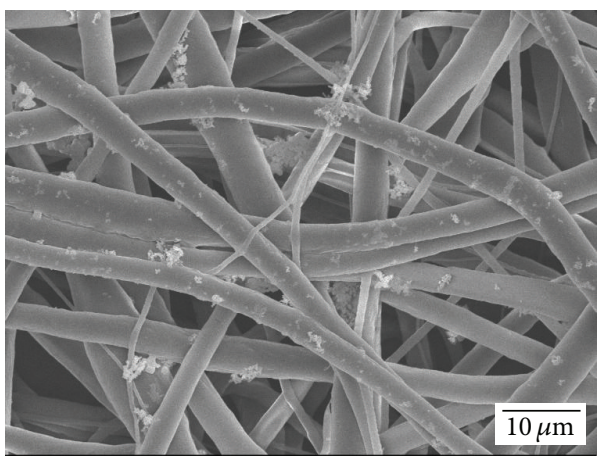

(a)

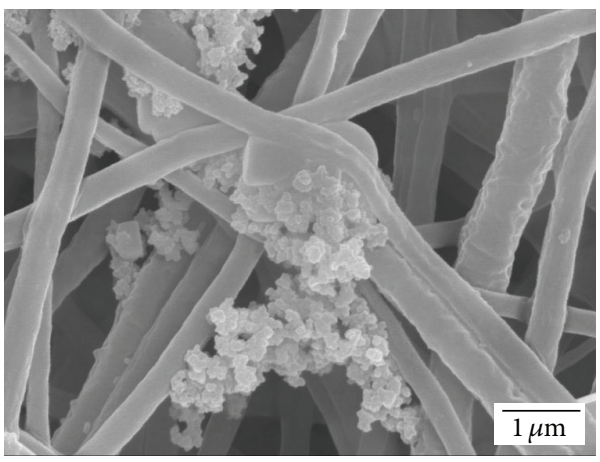

(c)

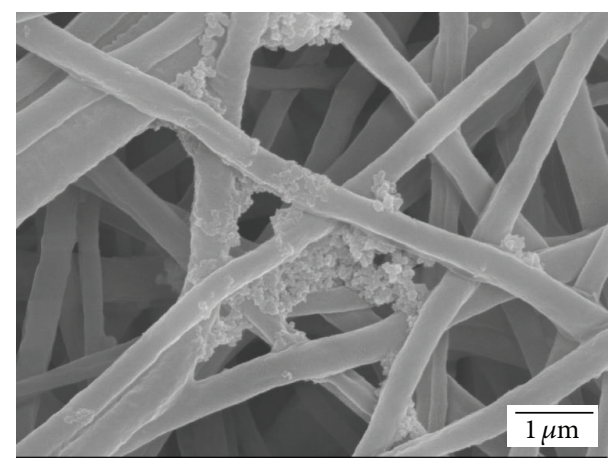

(b)

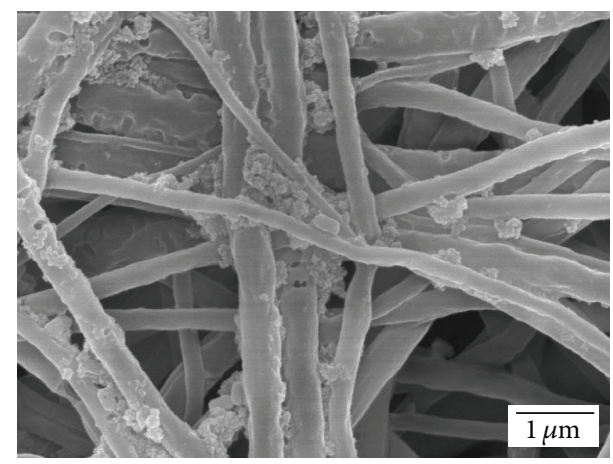

(d)

FIGURE 14: FE-SEM images of the TCH-loaded PCL/silica hybrid nanofibrous scaffolds with various silica sol contents of (a) 0 vol\%, (b) $10 \mathrm{vol} \%$, (c) $15 \mathrm{vol} \%$, and (d) $20 \mathrm{vol} \%$ after immersion in the SBF for 7 days.

by hybridizing the PCL polymer with the sol-gel-derived silica phase [39].

3.7. Drug Release Behavior. The drug release behavior of the TCH-loaded PCL/silica hybrid nanofibrous scaffolds was evaluated in PBS buffer solution over a period of $50 \mathrm{~h}$. Figures 10(A)-10(D) show the cumulative drug-release profiles of the hybrid nanofibrous scaffolds with various silica sol contents ( $0 \mathrm{vol} \%, 10 \mathrm{vol} \%, 15 \mathrm{vol} \%$, and $20 \mathrm{vol} \%$ ). The PCL/silica hybrid nanofibrous scaffolds showed much higher release rate of the TCH than the PCL nanofibrous scaffold. This was presumably attributed to both an increase in hydrophilicity of the PCL/silica hybrid nanofibers and faster degradation behavior of the silica phase. In addition, it should be noted that the hybrid nanofibrous scaffolds would have much higher release rate than relatively dense hybrids owing to their high surface-to-volume ratio. The cumulative maximum amounts of the TCH released from the PCL/silica hybrid nanofibrous scaffolds with initial silica sol contents of 0 vol $\%, 10 \mathrm{vol} \%, 15 \mathrm{vol} \%$, and $20 \mathrm{vol} \%$ were $35 \pm 5 \%, 64 \pm 3 \%$, $82 \pm 3 \%$, and $80 \pm 4 \%$, respectively. As mentioned earlier, the $\mathrm{TCH}$ drug tended to be segregated from the PCL solution during electrospinning process, resulting in a lower loading efficiency of the TCH into the PCL nanofibers. However, the loading efficiency is considerably enhanced by hybridizing the PCL polymer with the silica phase.
3.8. In Vitro Biocompatibility. The in vitro biocompatibility of the TCH-loaded PCL/silica hybrid nanofibrous scaffolds was assessed by in vitro cell tests using MC3T3-E1 cells. Figures 11(a)-1(d) show typical CLSM images of the cells attached to the hybrid nanofibrous scaffolds with various silica sol contents ( $0 \mathrm{vol} \%, 10 \mathrm{vol} \%, 15 \mathrm{vol} \%$, and $20 \mathrm{vol} \%)$ after $6 \mathrm{~h}$ of culturing. The red and blue colors represent the actin and nucleus, respectively. Basically, all hybrid nanofibrous scaffolds showed that the cells are actively attached and spread on the surface, suggesting good biocompatibility.

The absorbance values, which represent the degree of cell proliferation, on the TCH-loaded PCL/silica hybrid nanofibrous scaffolds with various silica sol contents ( 0 vol\%, $10 \mathrm{vol} \%, 15 \mathrm{vol} \%$, and $20 \mathrm{vol} \%$ ) were examined after 1 and 2 days of culturing using an MTS assay, as shown in Figures 12(a) and 12(b). The hybrid nanofibrous scaffolds with silica contents of $0 \mathrm{vol} \%, 10 \mathrm{vol} \%$, and $15 \mathrm{vol} \%$ showed similar absorbance values after 1 day of culturing, while the scaffold with a highest silica content of $20 \mathrm{vol} \%$ showed a higher absorbance value (Figure 12(a)). In addition, the absorbance values increased remarkably after 2 days of culturing for all produced hybrid nanofibrous scaffolds (Figure 12(b)). This finding suggests that the loading of the TCH drug into PCL/silica hybrid nanofibrous scaffolds has no negative effects on the growth of osteoblasts, allowing for good biocompatibility [40]. 
The continued cell proliferation on the TCH-loaded PCL/silica hybrid nanofibrous scaffolds was evaluated after 7 days of culturing, as shown in Figures 13(a)-13(d). The hybrid nanofibrous scaffolds with higher silica contents ( 15 vol\% and $20 \mathrm{vol} \%$ ) showed more active extensions and bridging of extracellular matrix (ECM) (Figures 13(c) and 13(d)), representing the early stage of mineralization of MC3T3-E1 cells [41]. It should be noted that TCH-loaded PCL/silica hybrid nanofibrous scaffolds would show excellent bone regeneration in vivo, as is often the case with nanoporous $\mathrm{PCL} /$ silica xerogel membranes [39].

3.9. In Vitro Apatite-Forming Ability. The in vitro mineralization behavior of the TCH-loaded PCL/silica hybrid nanofibrous scaffolds, one of the most important key markers for representing the ability to induce new bone tissue formation in vivo, was more closely evaluated using SBF solution. Figures 14(a)-14(b) show typical SEM images of the hybrid nanofibrous scaffolds with various silica contents $(0 \mathrm{vol} \%$, 10 vol\%, 15 vol\%, and 20 vol\%) after immersion in the SBF solution for 7 days. Compared to the PCL scaffold, the hybrid scaffolds showed more vigorous precipitation of apatite crystals, suggesting significantly enhanced mineralization ability owing to the excellent apatite-forming ability of the silica phase.

\section{Conclusions}

TCH-loaded PCL/silica hybrid nanofibrous scaffolds with various silica sol contents ( $10 \mathrm{vol} \%, 15 \mathrm{vol} \%$, and $20 \mathrm{vol} \%$ ) were successfully produced using ES. The silica phase was homogenously hybridized with the PCL polymer, while their intrinsic characteristics were preserved. This significantly improved the hydrophilic nature and mechanical properties of the hybrid nanofibrous scaffolds. The hybrid nanofibrous scaffold with an initial silica sol content of $15 \mathrm{vol} \%$ had reasonably high ultimate tensile strength of $5.5 \pm 0.3 \mathrm{MPa}$ and elastic modulus of $19.8 \pm 2.9 \mathrm{MPa}$ as well as a low contact angle of $64 \pm 3^{\circ}$. In addition, the hybrid nanofibrous scaffolds showed much higher release rate and maximum amounts of the TCH than the PCL nanofibrous scaffold. Regardless of the silica content, all produced hybrid nanofibrous scaffolds showed biocompatibility assessed in terms of the proliferation of pre-osteoblast cells. These findings suggest that TCH-loaded $\mathrm{PCL} /$ silica hybrid nanofibrous scaffolds have great potential as a membrane for guided bone regeneration (GBR).

\section{Conflict of Interests}

The authors declare that there are no conflicts of interest.

\section{Acknowledgment}

This research was supported by Korea Healthcare technology R\&D Project, Ministry of Health and Welfare, Republic of Korea (Contract Grant no. HI11C0388).

\section{References}

[1] M. M. Stevens and J. H. George, "Exploring and engineering the cell surface interface," Science, vol. 310, no. 5751, pp. 1135-1138, 2005.

[2] S. Gronthos, P. J. Simmons, S. E. Graves, and P. G. Robey, "Integrin-mediated interactions between human bone marrow stromal precursor cells and the extracellular matrix," Bone, vol. 28, no. 2, pp. 174-181, 2001.

[3] M. P. Lutolf and J. A. Hubbell, "Synthetic biomaterials as instructive extracellular microenvironments for morphogenesis in tissue engineering," Nature Biotechnology, vol. 23, no. 1, pp. 47-55, 2005.

[4] M. Schindler, I. Ahmed, J. Kamal et al., "A synthetic nanofibrillar matrix promotes in vivo-like organization and morphogenesis for cells in culture," Biomaterials, vol. 26, no. 28, pp. 5624-5631, 2005.

[5] D. Ishii, T. H. Ying, A. Mahara et al., "In vivo tissue response and degradation behavior of PLLA and stereocomplexed PLA nanofibers," Biomacromolecules, vol. 10, no. 2, pp. 237-242, 2009.

[6] W. Teo, W. He, and S. Ramakrishna, "Electrospun scaffold tailored for tissue-specific extracellular matrix," Biotechnology Journal, vol. 1, no. 9, pp. 918-929, 2006.

[7] S. Agarwal, J. H. Wendorff, and A. Greiner, "Progress in the field of electrospinning for tissue engineering applications," Advanced Materials, vol. 21, no. 32-33, pp. 3343-3351, 2009.

[8] J. H. Jang, O. Castano, and H. W. Kim, "Electrospun materials as potential platforms for bone tissue engineering," Advanced Drug Delivery Reviews, vol. 61, no. 12, pp. 1065-1083, 2009.

[9] E. R. Kenawy, G. L. Bowlin, K. Mansfield et al., "Release of tetracycline hydrochloride from electrospun poly(ethyleneco-vinylacetate), poly(lactic acid), and a blend," Journal of Controlled Release, vol. 81, no. 1-2, pp. 57-64, 2002.

[10] P. Taepaiboon, U. Rungsardthong, and P. Supaphol, "Drugloaded electrospun mats of poly(vinyl alcohol) fibres and their release characteristics of four model drugs," Nanotechnology, vol. 17, no. 9, pp. 2317-2329, 2006.

[11] K. Kanawung, K. Panitchanapan, S. Puangmalee et al., "Preparation and characterization of polycaprolactone/ diclofenac sodium and poly(vinyl alcohol)/tetracycline hydrochloride fiber mats and their release of the model drugs," Polymer Journal, vol. 39, no. 4, pp. 369-378, 2007.

[12] Z. X. Meng, W. Zheng, L. Li, and Y. F. Zheng, "Fabrication, characterization and in vitro drug release behavior of electrospun PLGA/chitosan nanofibrous scaffold," Materials Chemistry and Physics, vol. 125, no. 3, pp. 606-611, 2011.

[13] P. Zahedi, Z. Karami, I. Rezaeian et al., "Preparation and performance evaluation of tetracycline hydrochloride loaded wound dressing mats based on electrospun nanofibrous poly(lactic acid)/poly( $\varepsilon \mu$-caprolactone) blends," Journal of Applied Polymer Science, vol. 124, no. 5, pp. 4174-4183, 2012.

[14] L. A. Mary, T. Senthilram, S. Suganya et al., "Centrifugal spun ultrafine fibrous web as a potential drug delivery vehicle," Express Polymer Letters, vol. 7, pp. 238-248, 2013.

[15] A. M. Le Ray, S. Chiffoleau, P. Iooss et al., "Vancomycin encapsulation in biodegradable poly( $\varepsilon$-caprolactone) microparticles for bone implantation. Influence of the formulation process on size, drug loading, in vitro release and cytocompatibility," Biomaterials, vol. 24, no. 3, pp. 443-449, 2003.

[16] L. Meseguer-Olmo, M. J. Ros-Nicolás, M. Clavel-Sainz et al., "Biocompatibility and in vivo gentamicin release from bioactive 
sol-gel glass implants," Journal of Biomedical Materials Research, vol. 61, no. 3, pp. 458-465, 2002.

[17] W. J. Li, K. G. Danielson, P. G. Alexander, and R. S. Tuan, "Biological response of chondrocytes cultured in three-dimensional nanofibrous poly( $\varepsilon$-caprolactone) scaffolds," Journal of Biomedical Materials Research A, vol. 67, no. 4, pp. 1105-1114, 2003.

[18] H. Yoshimoto, Y. M. Shin, H. Terai, and J. P. Vacanti, "A biodegradable nanofiber scaffold by electrospinning and its potential for bone tissue engineering," Biomaterials, vol. 24, no. 12, pp. 2077-2082, 2003.

[19] M. Shin, H. Yoshimoto, and J. P. Vacanti, "In vivo bone tissue engineering using mesenchymal stem cells on a novel electrospun nanofibrous scaffold," Tissue Engineering, vol. 10, no. 1-2, pp. 33-41, 2004.

[20] M. S. Khil, S. R. Bhattarai, H. Y. Kim, S. Kim, and K. Lee, "Novel fabricated matrix via electrospinning for tissue engineering," Journal of Biomedical Materials Research B, vol. 72, no. 1, pp. 117124, 2005.

[21] K. Fujihara, M. Kotaki, and S. Ramakrishna, "Guided bone regeneration membrane made of polycaprolactone/calcium carbonate composite nano-fibers," Biomaterials, vol. 26, no. 19, pp. 4139-4147, 2005.

[22] P. Wutticharoenmongkol, N. Sanchavanakit, P. Pavasant, and P. Supaphol, "Novel bone scaffolds of electrospun polycaprolactone fibers filled with nanoparticles," Journal of Nanoscience and Nanotechnology, vol. 6, no. 2, pp. 514-522, 2006.

[23] C. Erisken, D. M. Kalyon, and H. Wang, "Functionally graded electrospun polycaprolactone and $\beta$-tricalcium phosphate nanocomposites for tissue engineering applications," Biomaterials, vol. 29, no. 30, pp. 4065-4073, 2008.

[24] E. M. Valliant and J. R. Jones, "Softening bioactive glass for bone regeneration: sol-gel hybrid materials," Soft Matter, vol. 7, no. 11, pp. 5083-5095, 2011.

[25] E. J. Lee, S. H. Teng, T. S. Jang et al., "Nanostructured poly( $\varepsilon$ caprolactone)-silica xerogel fibrous membrane for guided bone regeneration," Acta Biomaterialia, vol. 6, no. 9, pp. 3557-3565, 2010.

[26] T. S. Jang, E. J. Lee, J. H. Jo et al., "Fibrous membrane of nano-hybrid poly-L-lactic acid/silica xerogel for guided bone regeneration," Journal of Biomedical Materials Research B, vol. 100, no. 2, pp. 321-330, 2012.

[27] B. Lei, K. H. Shin, D. Y. Noh et al., "Nanofibrous gelatinsilica hybrid scaffolds mimicking the native extracellular matrix (ECM) using thermally induced phase separation," Journal of Materials Chemistry, vol. 22, pp. 14133-14140, 2012.

[28] Y. Wu, C. Wu, Y. Li, T. Xu, and Y. Fu, "PVA-silica anionexchange hybrid membranes prepared through a copolymer crosslinking agent," Journal of Membrane Science, vol. 350, no. 1-2, pp. 322-332, 2010.

[29] S. Cheng, D. Shen, X. Zhu, X. Tian, D. Zhou, and L. Fan, "Preparation of nonwoven polyimide/silica hybrid nanofiberous fabrics by combining electrospinning and controlled in situ sol-gel techniques," European Polymer Journal, vol. 45, no. 10, pp. 2767-2778, 2009.

[30] D. Cao, Y. P. Wu, Z. F. Fu et al., "Cell adhesive and growth behavior on electrospun nanofibrous scaffolds by designed multifunctional composites," Colloids and Surfaces B, vol. 84, no. 1, pp. 26-34, 2011.

[31] R. R. Duling, R. B. Dupaix, N. Katsube, and J. Lannutti, "Mechanical characterization of electrospun polycaprolactone (PCL): a potential scaffold for tissue engineering," Journal of
Biomechanical Engineering, vol. 130, no. 1, Article ID 011006, 2008.

[32] V. Pillay, C. Dott, Y. E. Choonara et al., "A review of the effect of processing variables on the fabrication of electrospun nanofibers for drug delivery applications," Journal of Nanomaterials, vol. 2013, Article ID 789289, 22 pages, 2013.

[33] A. Mohamed, V. L. Finkenstadt, S. H. Gordon, G. Biresaw, E. Palmquist Debra, and P. Rayas-Duarte, "Thermal properties of PCL/gluten bioblends characterized by TGA, DSC, SEM, and infrared-PAS," Journal of Applied Polymer Science, vol. 110, no. 5, pp. 3256-3266, 2008.

[34] S. Sayyar, E. Murray, B. C. Thompson, S. Gambhir, D. L. Officer, and G. G. Wallace, "Covalently linked biocompatible graphene/polycaprolactone composites for tissue engineering," Carbon, vol. 52, pp. 296-304, 2013.

[35] J. L. E. Ivirico, M. S. Sánchez, R. Sabater i Serra, J. M. M. Dueñas, J. L. G. Ribelles, and M. M. Pradas, "Structure and properties of poly( $\varepsilon$-caprolactone) networks with modulated water uptake," Macromolecular Chemistry and Physics, vol. 207, no. 23, pp. 2195-2205, 2006.

[36] D. Verma, K. Katti, and D. Katti, "Bioactivity in in situ hydroxyapatite-polycaprolactone composites," Journal of Biomedical Materials Research A, vol. 78, no. 4, pp. 772-780, 2006.

[37] S. H. Jun, E. J. Lee, S. W. Yook, H. E. Kim, H. W. Kim, and Y. H. Koh, "A bioactive coating of a silica xerogel/chitosan hybrid on titanium by a room temperature sol-gel process," Acta Biomaterialia, vol. 6, no. 1, pp. 302-307, 2010.

[38] A. Fidalgo and L. M. Ilharco, "The influence of the wet gels processing on the structure and properties of silica xerogels," Microporous and Mesoporous Materials, vol. 84, no. 1-3, pp. 229235, 2005.

[39] E. J. Lee, S. H. Teng, T. S. Jang et al., "Nanostructured poly( $(\varepsilon-$ caprolactone)-silica xerogel fibrous membrane for guided bone regeneration," Acta Biomaterialia, vol. 6, no. 9, pp. 3557-3565, 2010.

[40] L. F. Zhang, R. Sun, L. Xu et al., "Hydrophilic poly (ethylene glycol) coating on PDLLA/BCP bone scaffold for drug delivery and cell culture," Materials Science and Engineering C, vol. 28, no. 1, pp. 141-149, 2008.

[41] J. P. St-Pierre, M. Gauthier, L. P. Lefebvre, and M. Tabrizian, "Three-dimensional growth of differentiating MC3T3-E1 preosteoblasts on porous titanium scaffolds," Biomaterials, vol. 26, no. 35, pp. 7319-7328, 2005. 

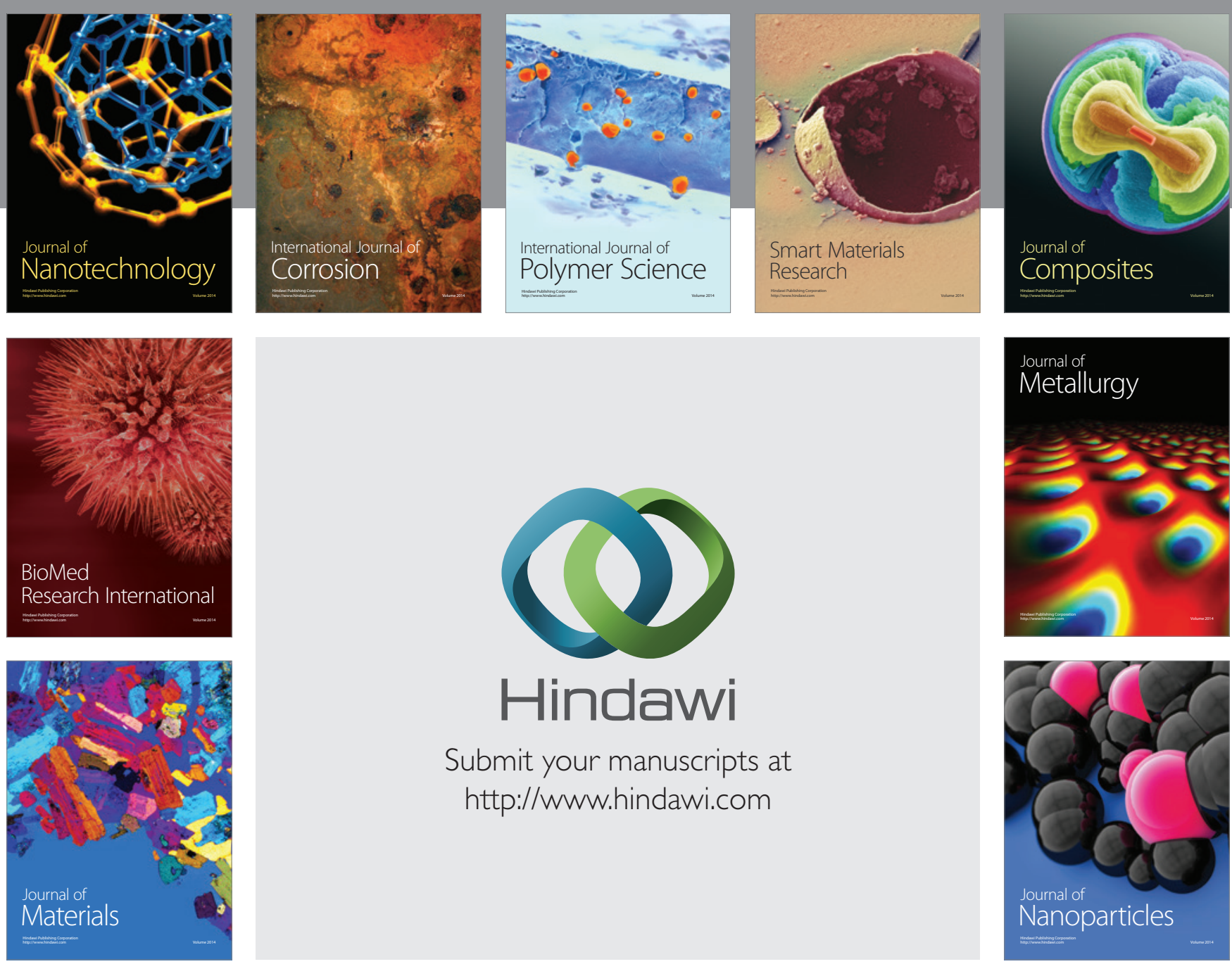

Submit your manuscripts at http://www.hindawi.com
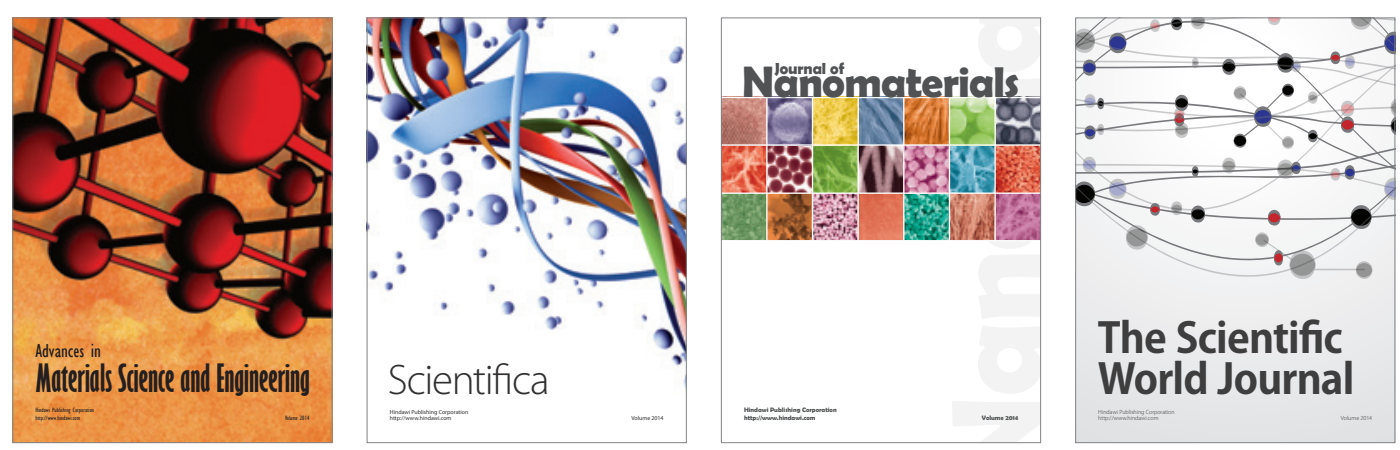

\section{The Scientific World Journal}
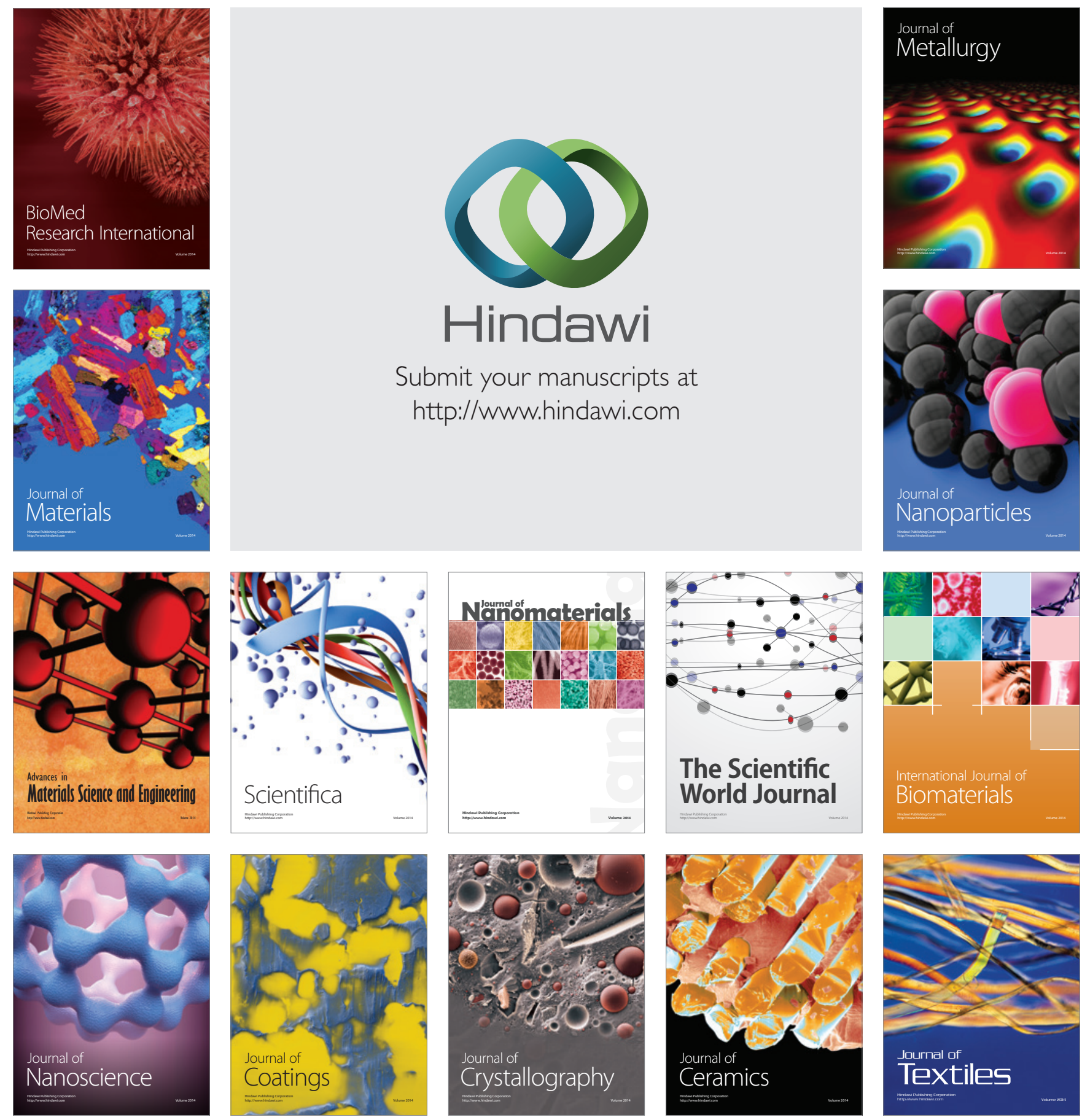